Bull. Austral. Math. Soc.

Vol. 74 (2006) [45-68]

\title{
PARALLEL METRICS AND REDUCIBILITY OF THE HOLONOMY GROUP
}

\author{
RICHARD ATKINS
}

In this paper we investigate the relationship between the existence of parallel semiRiemannian metrics of a connection and the reducibility of the associated holonomy group. The question as to whether the holonomy group necessarily reduces in the presence of a specified number of independent parallel semi-Riemannian metrics is completely determined by the the signature of the metrics and the dimension $d$ of the manifold, when $d \neq 4$. In particular, the existence of two independent, parallel semi-Riemannian metrics, one of which having signature $(p, q)$ with $p \neq q$, implies the holonomy group is reducible. The $(p, p)$ cases, however, may allow for more than one parallel metric and yet an irreducible holonomy group: for $n=2 m, m \geqslant 3$, there exist connections on $R^{n}$ with irreducible infinitesimal holonomy and which have two independent, parallel metrics of signature $(m, m)$. The case of four-dimensional manifolds, however, depends on the topology of the manifold in question: the presence of three parallel metrics always implies reducibility but reducibility in the case of two metrics of signature $(2,2)$ is guaranteed only for simply connected manifolds. The main theorem in the paper is the construction of a topologically non-trivial four-dimensional manifold with a connection that admits two independent metrics of signature $(2,2)$ and yet has irreducible holonomy. We provide a complete solution to the general problem.

\section{INTRODUCTION}

This paper investigates the relationship between two fundamental types of objects associated with a connection on a manifold: the existence of parallel semi-Riemannian metrics and the associated holonomy group. Typically in Riemannian geometry a metric is specified, which determines a Levi-Civita connection. Here we consider the connection as more fundamental and allow for the possibility of several parallel metrics. Holonomy is an old geometric concept, which is enjoying revived interest in certain branches of mathematical physics, in particular loop quantum gravity and Calabi-Yau manifolds in string theory. It measures, in group theoretic terms the connection's deviation from flatness and takes the topology of the manifold into account.

Received 22nd February, 2006

Copyright Clearance Centre, Inc. Serial-fee code: 0004-9727/06 \$A2.00+0.00. 
It is well known that for a Riemannian manifold, the reducibility of the holonomy group of the Levi-Civita connection implies the existence of multiple independent parallel Riemannian metrics on the manifold [5]. In this paper we look at the converse: when does the existence of multiple independent semi-Riemannian metrics on a manifold, parallel with respect to a linear connection, imply the reducibility of the holonomy group of the connection? We do not necessarily assume that the connection is symmetric in our solutions to this problem. If $g$ is a semi-Riemannian metric on $M$, which is parallel with respect to the connection $\nabla$, then the holonomy group $\Psi(x)$, at $x \in M$, preserves $g: \psi^{*}(g)=g$, for all $\psi \in \Psi(x)$. Thus the existence of parallel metrics places algebraic restrictions on $\Psi(x)$; these restrictions will be the subject of our investigations. For manifolds of dimension $d \neq 4$ the problem has a purely algebraic solution. For fourdimensional manifolds the relationship of the parallel metrics of the connection to the reducibility of the holonomy group is not entirely algebraic but depends also on the fundamental group of the manifold: the presence of three parallel metrics always implies reducibility but reducibility in the case of two metrics of signature $(2,2)$ is guaranteed only for simply connected manifolds. The central theorem in this paper is the construction of a topologically non-trivial four-dimensional manifold with a connection that admits two independent parallel metrics of signature $(2,2)$ and yet has irreducible holonomy. $d=4$ is the critical dimension with respect to reducibility of the holonomy group.

It is interesting to note that $d=4$ appears as the critical dimension in other contexts as well. In quantum field theory, for instance, infinite divergences appear in the calculation of scattering amplitudes as the dimension of spacetime approaches four. Also, it has been shown that $R^{4}$ has the remarkable property of admitting exotic differentiable structures $[1,2]$. In superstring theory spacetime is ten or eleven dimensional but only four dimensions are observed in nature. Therefore some unique characteristic of fourdimensional manifolds must be involved in explaining this mismatch of dimensions.

In Section 2 we state the main results of the paper. Section 3 gives the proofs relating to the reducibility of the holonomy group for $d \neq 4$. Section 4 provides proof of the existence of connections with irreducible holonomy in the presence of parallel metrics for $d \neq 4$. The final section deals with four-dimensional manifolds.

The problem of non-uniqueness of parallel metrics, largely for Lorentzian connections, has been investigated by several authors $[3,4,6,7]$.

\section{Statement of Results}

Let $V$ be a vector space over a field $F$, which will be either $R$ or $C$. Let $G$ be a group which acts on $V$ on the left. A subspace $W$ of $V$ is said to be $G$-invariant if $g \cdot \xi \in W$, for all $g \in G$ and $\xi \in W$. If there exists a proper, non-trivial $G$-invariant subspace of $V$ we say that $G$ acts reducibly on $V$, or more simply, that $G$ is reducible. In our applications, $G$ shall be a subgroup of $\operatorname{Aut}(V)$, the group of linear automorphisms of $V$. The holonomy 
group $\Psi(x)$ of a linear connection $\nabla$ on a connected manifold $M$, at $x \in M$, is a subgroup of Aut $\left(T_{x} M\right)$. For any two points $x, y \in M$, the holonomy groups $\Psi(x)$ and $\Psi(y)$ are isomorphic, since $M$ is connected. If for some point (and hence all points) $x \in M, \Psi(x)$ is reducible (respectively, irreducible) then we say that the connection $\nabla$ has reducible (respectively, irreducible) holonomy.

We begin with a theorem that provides sufficient conditions, with regard to the existence of parallel semi-Riemannian metrics, to ensure the reducibility of the holonomy group of the connection.

THEOREM 2.0.1. (i) Let $g_{1}$ and $g_{2}$ be two independent semi-Riemannian metrics on a connected manifold $M$, parallel with respect to a linear connection $\nabla$ on $M$. Suppose that one of $g_{1}, g_{2}$ has signature $(p, q)$ with $p \neq q$. Then $\nabla$ has reducible holonomy.

(ii) Let $g_{1}$ and $g_{2}$ be two independent semi-Riemannian metrics on a connected, two-dimensional manifold $M$, parallel with respect to a linear connection $\nabla$ on $M$. Then $\nabla$ has reducible holonomy.

(iii) Suppose $n \not \equiv 0$ mod 4 . Let $g_{1}, g_{2}$ and $g_{3}$ be three independent semi-Riemannian metrics on a connected, $n$-dimensional manifold $M$, parallel with respect to a linear connection $\nabla$ on $M$. Then $\nabla$ has reducible holonomy.

(iv) Let $g_{1}, g_{2}, g_{3}$ and $g_{4}$ be four independent semi-Riemannian metrics on a connected manifold $M$, parallel with respect to a linear connection $\nabla$ on $M$. Then $\nabla$ has reducible holonomy.

It is possible to construct examples of connections on manifolds that show that the numbers of parallel semi-Riemannian metrics in the above theorem are sharp. Specifically we have the following.

THEOREM 2.0.2. (i) Let $n=2 m$ and $m \geqslant 3$. There exist two independent semi-Riemannian metrics on $R^{n}$ of signature $(m, m)$, parallel with respect to a linear connection having an irreducible holonomy group.

(ii) Let $n=2 m, m=2 r$ and $r \geqslant 2$. There exist three independent semiRiemannian metrics on $R^{n}$ of signature $(m, m)$, parallel with respect to a linear connection having an irreducible holonomy group.

In four dimensions we have the following results.

THEOREM 2.0.3. (i) Let $M$ be a connected, simply connected, four-dimensional manifold. Let $g_{1}$ and $g_{2}$ be two independent semi-Riemannian metrics on $M$, parallel with respect to a linear connection $\nabla$ on $M$. Then $\nabla$ has reducible holonomy.

(ii) Let $M$ be a connected, four-dimensional manifold. Let $g_{1}, g_{2}$ and $g_{3}$ be three independent semi-Riemannian metrics on $M$, parallel with respect to a linear connection $\nabla$ on $M$. Then $\nabla$ has reducible holonomy.

(iii) There exists a connected, non-simply connected, four-dimensional manifold $M$ 
with two independent semi-Riemannian metrics of signature $(2,2)$, parallel with respect to a linear connection $\nabla$ on $M$, where $\nabla$ has irreducible holonomy.

\section{Proof of Theorem 2.0.1}

$V$ shall always denote an $n$-dimensional vector space. If $n$ is even then we set $n=2 m$ and if $m$ is even we set $m=2 r$. When $n$ is even, $K$ and $L$ shall always denote the matrices in $G L(n ; R)$ defined by

$$
K:=\left(\begin{array}{cc}
I_{m \times m} & 0 \\
0 & -I_{m \times m}
\end{array}\right) \text { and } L:=\left(\begin{array}{cc}
0 & I_{m \times m} \\
I_{m \times m} & 0
\end{array}\right)
$$

$K$ and $L$ may be regarded as symmetric bilinear forms on $R^{n}$ with signature $(m, m)$.

Proofs of the less technical lemmas and corollaries shall be left to the reader in what follows.

3.1. TWO SYMMETRIC BILINEAR FORMS. In this subsection we examine the case of a subgroup of $\operatorname{Aut}(V)$ preserving two independent, symmetric, non-degenerate bilinear forms on $V$.

Let $G$ be a subgroup of $G L(n ; F) . G$ acts on $V:=F^{n}$ on the left by matrix multiplication:

$$
g \cdot \xi=g \xi
$$

for all $g \in G$ and $\xi \in F^{n}$. The essence of the following lemma may be found in [5, p. 277].

LEMMA 3.1.1. Let $G$ be a subgroup of $G L(n ; F)$ which acts irreducibly on $F^{n}$ and let $A \in g l(n ; F)$ commute with each element of $G$.

(1) The minimal polynomial of $A$ (over $F$ ) is irreducible (over $F$ ).

(2) Let $F=R$.

(i) $A=a I$, for some $a \in R$, when $n$ is odd, and

(ii) $A=a I+b J$, for some $a, b \in R$ and $J \in G L(n ; R)$, which satisfies $J^{2}=-I$, when $n$ is even.

Let $\eta_{1}$ and $\eta_{2}$ be two independent, symmetric, non-degenerate bilinear forms on $V$ and let $\eta_{1}$ have signature $(p, q)$. Let $\mathcal{G}$ be a subgroup of the automorphism group of $V$ whose elements preserve $\eta_{1}$ and $\eta_{2}: g^{*}\left(\eta_{i}\right)=\eta_{i}$, for all $g \in \mathcal{G}$ and $i=1,2$. Let $K_{p, q} \in G L(n ; R)$ be defined by

$$
K_{p, q}:=\left(\begin{array}{cc}
I_{p \times p} & 0 \\
0 & -I_{q \times q}
\end{array}\right)
$$

LEMMA 3.1.2. Suppose that $\mathcal{G}$ acts irreducibly on $V$. Let $\beta$ be any basis in which $\eta_{1}$ is represented by $K_{p, q}$. Then in this basis $\eta_{2}$ is represented by $a K_{p, q}+b K_{p, q} J$, where $a, b \in R$ and $J \in G L(n ; R)$ satisfies $J^{2}=-I$. 
Proof: Let $\beta$ be a basis of $V$ in which $\eta_{1}$ has the form $K_{p, q} . \eta_{2}$ will be represented by a symmetric matrix $A \in G L(n ; R)$, with respect to $\beta$. $\mathcal{G}$ may be identified with a subgroup $\mathcal{H}$ of $G L(n ; R)$ whose elements $H \in \mathcal{H}$ satisfy

$$
\left\{\begin{array}{l}
{ }^{t} H K_{p, q} H=K_{p, q}, \text { and } \\
{ }^{t} H A H=A .
\end{array}\right.
$$

Now ${ }^{t} H=K_{p, q} H^{-1} K_{p, q}$, for each $H \in \mathcal{H}$. Therefore, $A={ }^{t} H A H=K_{p, q} H^{-1} K_{p, q} A H$. That is,

$$
H\left(K_{p, q} A\right)=\left(K_{p, q} A\right) H,
$$

for all $H \in \mathcal{H}$. By Lemma 3.1.1, we see that $n$ must be even and that $K_{p, q} A=a I+b J$, where $a, b \in R$ and $J \in G L(n ; R)$ satisfies $J^{2}=-I$. Thus $A=a K_{p, q}+b K_{p, q} J$.

LEMMA 3.1.3. Suppose that one of $\eta_{1}, \eta_{2}$ has signature $(s, t)$ with $s \neq t$. Then $\mathcal{G}$ acts reducibly on $V$.

Proof: Suppose $\mathcal{G}$ acts irreducibly on $V$. Without loss of generality we may suppose that $p \neq q$. By Lemma 3.1.2, we have a basis of $V$ in which $\eta_{1}$ is represented by $K_{p, q}$ and $\eta_{2}$ is represented by $A=a K_{p, q}+b K_{p, q} J$, where $a, b \in R$ and $J^{2}=-I$. Since $A$ and $K_{p, q}$ are symmetric, $K_{p, q} J$ is symmetric. We have

$$
{ }^{t} J K_{p, q} J={ }^{t}\left(K_{p, q} J\right) J=K_{p, q} J J=-K_{p, q} .
$$

Now, the signature of $K_{p, q}$ is $(p, q)$ and must be equal to the signature $(q, p)$ of ${ }^{t} J K_{p, q} J$ $=-K_{p, q}$. Therefore $p=q$. This is a contradiction.

This proves Theorem 2.0.1 (i).

3.2. Two SYMMETRIC BILINEAR FORMS OF SIGNATURE $(m, m)$. Let $\eta_{1}$ and $\eta_{2}$ be two independent, symmetric bilinear forms on $V$ of signature $(m, m)$, where $n=2 m$. Let $\mathcal{G}$ denote a subgroup of $\operatorname{Aut}(V)$ preserving $\eta_{1}$ and $\eta_{2}$. In this subsection we find a normal form for $\eta_{1}$ and $\eta_{2}$ when $\mathcal{G}$ acts irreducibly on $V$.

LEMMA 3.2.1. Suppose that $\mathcal{G}$ acts irreducibly on $V$. Then there exists a basis $\beta$ of $V$ in which $\eta_{1}$ is represented by $K$ and $\eta_{2}$ is represented by $A=a K+b K J$ where $a, b \in R, J^{2}=-I$ and $J$ has the form

$$
J=\left(\begin{array}{cc}
D_{1} & T \\
-^{t} T & -D_{2}
\end{array}\right),
$$

where $D_{1}$ and $D_{2}$ are diagonal matrices.

ProOF: Follows from Lemma 3.1.2 and a standard diagonalisation argument.

Let $M$ be an $m \times m$ matrix. With respect to $M$, we define two equivalence relations $\sim^{M, 1}$ and $\sim^{M, 2}$ on $\{1, \ldots, m\}$ as follows. $i \sim^{M, 1} j$ if and only if $i=j$ or there exists a sequence of non-zero elements

$$
M_{q_{1}}^{i}, M_{q_{1}}^{p_{1}}, M_{q_{2}}^{p_{1}}, M_{q_{2}}^{p_{2}}, M_{q_{3}}^{p_{2}}, M_{q_{3}}^{p_{3}}, \ldots, M_{q_{k}}^{p_{k-1}}, M_{q_{k}}^{j} .
$$


We set $i \sim \sim^{M, 2} j$ if and only if $i=j$ or there exists a sequence of non-zero elements

$$
M_{i}^{p_{1}}, M_{q_{1}}^{p_{1}}, M_{q_{1}}^{p_{2}}, M_{q_{2}}^{p_{2}}, M_{q_{2}}^{p_{3}}, M_{q_{3}}^{p_{3}}, \ldots, M_{q_{k-1}}^{p_{k}}, M_{j}^{p_{k}}
$$

We shall denote by $[i]^{M, 1}$ (respectively, $[i]^{M, 2}$ ) the elements which are $\sim^{M, 1}$ (respectively, $\sim^{M, 2}$ ) equivalent to $i$.

LEMMA 3.2.2. Suppose that $M_{j}^{i} \neq 0$.

(1) Let $p \in[i]^{M, 1}$ and $q \notin[j]^{M, 2}$. Then $M_{q}^{p}=0$.

(2) Let $p \notin[i]^{M, 1}$ and $q \in[j]^{M, 2}$. Then $M_{q}^{p}=0$.

LEMMA 3.2.3. Suppose that $\mathcal{G}$ acts irreducibly on $V$. Then there exists a basis $\beta$ of $V$ in which $\eta_{1}$ is represented by $K$ and $\eta_{2}$ is represented by $A=a K+b K J$, where $a, b \in R, J^{2}=-I$ and $J$ has the form

$$
J=\left(\begin{array}{cc}
D & T \\
-{ }^{t} T & -D
\end{array}\right),
$$

where $D$ is a diagonal matrix and $T$ is a block diagonal matrix of the form

$$
D=\left(\begin{array}{cccc}
a_{1} I & 0 & \cdots & 0 \\
0 & a_{2} I & \ddots & \vdots \\
\vdots & \ddots & \ddots & 0 \\
0 & \cdots & 0 & a_{\lambda} I
\end{array}\right) \text { and } T=\left(\begin{array}{cccc}
B_{1} & 0 & \cdots & 0 \\
0 & B_{2} & \ddots & \vdots \\
\vdots & \ddots & \ddots & 0 \\
0 & \cdots & 0 & B_{\lambda}
\end{array}\right)
$$

and the blocks in $D$ corresponding to those in $T$ have the same dimension.

ProOf: By Lemma 3.2.1, there exists a basis $\beta$ of $V$ whereby $\eta_{1}$ and $\eta_{2}$ are represented, respectively, by $K$ and $A=a K+b K J$ where $a, b \in R, J^{2}=-I$ and $J$ has the form

$$
J=\left(\begin{array}{cc}
D_{1} & T \\
-{ }^{t} T & -D_{2}
\end{array}\right),
$$

where $D_{1}$ and $D_{2}$ are diagonal matrices.

The equation $J^{2}=-I$ gives $T^{t} T=D_{1}^{2}+I$. Thus $(\operatorname{det} T)^{2}=\operatorname{det}\left(D_{1}^{2}+I\right)>0$. Therefore $T$ is invertible. Let $\left[i_{1}\right]^{T, 1}, \ldots,\left[i_{\lambda}\right]^{T, 1}$ be the $\sim^{T, 1}$ equivalence classes. For each $k \in\{1, \ldots, \lambda\}$, there exists a $j_{k} \in\{1, \ldots, m\}$ such that $T_{j_{k}}^{i_{k}} \neq 0$, since $T$ is invertible. By Lemma 3.2.2,

(1) for $p \in\left[i_{k}\right]^{T, 1}$ and $q \notin\left[j_{k}\right]^{T, 2}$, we have $T_{q}^{p}=0$, and

(2) for $p \notin\left[i_{k}\right]^{T, 1}$ and $q \in\left[j_{k}\right]^{T, 2}$, we have $T_{q}^{p}=0$.

Set

$$
\begin{aligned}
& \mu_{k}:=\operatorname{card}\left[i_{k}\right]^{T, 1}, \quad 1 \leqslant k \leqslant \lambda ; \quad \mu_{0}:=0, \quad \text { and } \\
& \nu_{k}:=\operatorname{card}\left[j_{k}\right]^{T, 2}, \quad 1 \leqslant k \leqslant \lambda ; \quad \nu_{0}:=0 .
\end{aligned}
$$


Let $q \in\{1, \ldots, m\}$. Since $T$ is invertible, there exists $p \in\{1, \ldots, m\}$ such that $T_{q}^{p} \neq 0$. Now, $p \in\left[i_{k}\right]^{T, 1}$ for some $k \in\{1, \ldots, \lambda\}$. It follows that $q \in\left[j_{k}\right]^{T, 2}$. Therefore every element $q \in\{1, \ldots, m\}$ belongs to one of the equivalence classes $\left[j_{k}\right]^{T, 2}$, with $k \in\{1, \ldots, \lambda\}$. That is, $\left[j_{1}\right]^{T, 2}, \ldots,\left[j_{\lambda}\right]^{T, 2}$ is a partition of $\{1, \ldots, m\}$. We have

$$
\mu_{0}+\cdots+\mu_{\lambda}=m=\nu_{0}+\cdots+\nu_{\lambda} .
$$

Let $P, Q \in O(m ; R)$ be the permutation matrices defined as follows. The $j^{\text {th }}$ column of a matrix $M$ will be denoted by $M_{j}$. Set

$$
\left(P_{\mu_{0}+\cdots+\mu_{k-1}+1}, \ldots, P_{\mu_{0}+\cdots+\mu_{k}}\right):=\left(e_{t_{1}}, \ldots, e_{t_{\mu_{k}}}\right),
$$

where $t_{1}<\cdots<t_{\mu_{k}}$ and $\left\{t_{1}, \ldots, t_{\mu_{k}}\right\}=\left[i_{k}\right]^{T, 1}$, for $1 \leqslant k \leqslant \lambda$. $e_{i}$ denotes the $i^{\text {th }}$ standard basis vector in $R^{m}$. Also,

$$
\left(Q_{\nu_{0}+\cdots+\nu_{k-1}+1}, \ldots, Q_{\nu_{0}+\cdots+\nu_{k}}\right):=\left(e_{t_{1}}, \ldots, e_{t_{\nu_{k}}}\right),
$$

where $t_{1}<\cdots<t_{\nu_{k}}$, and $\left\{t_{1}, \ldots, t_{\nu_{k}}\right\}=\left[j_{k}\right]^{T, 2}$, for $1 \leqslant k \leqslant \lambda$.

Set $S:={ }^{t} P T Q . S$ has a block diagonal form

$$
S=\left(\begin{array}{cccc}
B_{1} & 0 & \cdots & 0 \\
0 & B_{2} & \ddots & \vdots \\
\vdots & \ddots & \ddots & 0 \\
0 & \cdots & 0 & B_{\lambda}
\end{array}\right)
$$

where $B_{k}$ is a $\mu_{k} \times \nu_{k}$ matrix, $1 \leqslant k \leqslant \lambda$. Since $S$ is invertible we must have $\mu_{k}=\nu_{k}$ for all $1 \leqslant k \leqslant \lambda$, and so each $B_{k}$ is an invertible square matrix. Owing to the permutation matrices $P$ and $Q$, it is not difficult to see that the $\sim^{S, 1}$ and $\sim^{S, 2}$ equivalence classes are identical and are given by

$$
\begin{gathered}
\left\{1, \ldots, \mu_{1}\right\}, \\
\left\{\mu_{1}+1, \ldots, \mu_{1}+\mu_{2}\right\} \\
\left\{\mu_{1}+\mu_{2}+1, \ldots, \mu_{1}+\mu_{2}+\mu_{3}\right\}, \\
\vdots \\
\left\{\mu_{1}+\cdots+\mu_{\lambda-1}+1, \ldots, \mu_{1}+\cdots+\mu_{\lambda}\right\} .
\end{gathered}
$$

Define $F \in O(n ; R)$ by

$$
F:=\left(\begin{array}{ll}
P & 0 \\
0 & Q
\end{array}\right) .
$$

Let $\beta^{\prime}$ be the basis of $V$ defined by setting $\beta^{\prime}:=\beta F$. $\eta_{1}$ is again represented by $K$ and $\eta_{2}$ is represented by $A^{\prime}={ }^{t} F A F=a K+b K J^{\prime}$, where $J^{\prime}:={ }^{t} F J F$ satisfies $\left(J^{\prime}\right)^{2}=-I$. Moreover $J^{\prime}$ has the form

$$
J^{\prime}=\left(\begin{array}{cc}
E_{1} & S \\
-{ }^{t} S & -E_{2}
\end{array}\right),
$$


where $E_{1}={ }^{t} P D_{1} P$ and $E_{2}={ }^{t} Q D_{2} Q$. Since $P$ and $Q$ are permutation matrices, $E_{1}$ and $E_{2}$ are diagonal matrices. The equation $\left(J^{\prime}\right)^{2}=-I$ gives us $E_{1} S-S E_{2}=0$. Therefore,

$$
0=\left(E_{1} S-S E_{2}\right)_{j}^{i}=S_{j}^{i}\left(\left(E_{1}\right)_{i}^{i}-\left(E_{2}\right)_{j}^{j}\right),
$$

for all $i, j \in\{1, \ldots, m\}$. It follows that $\left(E_{1}\right)_{i}^{i}=\left(E_{2}\right)_{j}^{j}$ when $S_{j}^{i} \neq 0$. Hence

$$
\begin{array}{ll}
\left(E_{1}\right)_{i}^{i}=\left(E_{1}\right)_{j}^{j} & \text { when } i \sim^{S, 1} j, \text { and } \\
\left(E_{2}\right)_{i}^{i}=\left(E_{2}\right)_{j}^{j} & \text { when } i \sim^{S, 2} j .
\end{array}
$$

Therefore

$$
E_{1}=\left(\begin{array}{cccc}
a_{1} I & 0 & \cdots & 0 \\
0 & a_{2} I & \ddots & \vdots \\
\vdots & \ddots & \ddots & 0 \\
0 & \cdots & 0 & a_{\lambda} I
\end{array}\right) \text { and } E_{2}=\left(\begin{array}{cccc}
b_{1} I & 0 & \cdots & 0 \\
0 & b_{2} I & \ddots & \vdots \\
\vdots & \ddots & \ddots & 0 \\
0 & \cdots & 0 & b_{\lambda} I
\end{array}\right)
$$

for some $a_{i}, b_{i} \in R$. Since $E_{1} S-S E_{2}=0$ and each $B_{k}$ is an invertible square matrix, it follows that $E_{1}=E_{2}$. This proves the lemma.

Lemma 3.2.4. Suppose that $\mathcal{G}$ acts irreducibly on $V$. Then there exists a basis $\beta$ of $V$ in which $\eta_{1}$ is represented by $K$ and $\eta_{2}$ is represented by $A=a K+b K J$ where $a, b \in R, J^{2}=-I$ and $J$ has the form

$$
J=\left(\begin{array}{cc}
D & D^{\prime} \\
-D^{\prime} & -D
\end{array}\right),
$$

where $D$ and $D^{\prime}$ are diagonal matrices of the form

$$
D=\left(\begin{array}{cccc}
a_{1} I & 0 & \cdots & 0 \\
0 & a_{2} I & \ddots & \vdots \\
\vdots & \ddots & \ddots & 0 \\
0 & \cdots & 0 & a_{\lambda} I
\end{array}\right), \quad D^{\prime}=\left(\begin{array}{cccc}
\sqrt{a_{1}^{2}+1} I & 0 & \cdots & 0 \\
0 & \sqrt{a_{2}^{2}+1} I & \ddots & \vdots \\
\vdots & \ddots & \ddots & 0 \\
0 & \cdots & 0 & \sqrt{a_{\lambda}^{2}+1} I
\end{array}\right),
$$

and the blocks in $D$ corresponding to those in $D^{\prime}$ have the same dimension.

Proof: Follows from Lemma 3.2.3 and a standard diagonalisation argument.

We finally arrive at the following normal form for $\eta_{1}$ and $\eta_{2}$.

LEMMA 3.2.5. Let $\eta_{1}$ and $\eta_{2}$ be two independent, symmetric bilinear forms on $V$ of signature $(m, m)$, where $n=2 m$. Let $\mathcal{G}$ be a subgroup of $\operatorname{Aut}(V)$ whose elements preserve $\eta_{1}$ and $\eta_{2}$. Suppose that $\mathcal{G}$ acts irreducibly on $V$. Then there exists a basis of $V$ with respect to which $\eta_{1}$ and $\eta_{2}$ are represented, respectively, by $K$ and $a K+b L$, for some $a, b \in R$. 
Proof: By Lemma 3.2.4, there exists a basis $\beta$ of $V$ with respect to which $\eta_{1}$ is represented by $K$ and $\eta_{2}$ is represented by $A=a K+b K J$ where $J$ has the form

$$
J=\left(\begin{array}{cc}
D & D^{\prime} \\
-D^{\prime} & -D
\end{array}\right),
$$

where $D$ and $D^{\prime}$ are diagonal matrices as given by the lemma. Set $E:=D+D^{\prime} . E$ is a diagonal matrix with positive entries along the main diagonal. Let $T$ be the diagonal matrix with positive entries such that $T^{2}=E$. Define $F \in G L(n ; R)$ by

$$
F:=\frac{1}{2}\left(\begin{array}{ll}
T^{-1}+T & T^{-1}-T \\
T^{-1}-T & T^{-1}+T
\end{array}\right) .
$$

We define the basis $\beta^{\prime}$ of $V$ by $\beta^{\prime}=\beta F$. A calculation will verify that with respect to $\beta^{\prime}$, $\eta_{1}$ is represented by ${ }^{t} F K F=K$ and $\eta_{2}$ is represented by $A^{\prime}={ }^{t} F A F=a K+b L$.

3.3. COMPLEXIFICATION OF THE hOLONOMY GROUP. Let $\mathcal{F}$ be the subgroup of $G L(n ; R)$ whose elements $F$ are of the form

$$
F=\left(\begin{array}{cc}
A & B \\
-B & A
\end{array}\right)
$$

The map $\phi: \mathcal{F} \rightarrow G L(m ; C)$ defined by

$$
\phi(F):=A+i B
$$

is a group monomorphism. Observe that $\phi\left({ }^{t} F\right)={ }^{t} \overline{\phi(F)}$, for all $F \in \mathcal{F}$, where the bar denotes complex conjugation. Let $\mathcal{G}$ be any subgroup of $\mathcal{F}$. We denote by $\mathcal{G}_{C}$ the subgroup $\phi(\mathcal{G})$ of $G L(m ; C)$. $\phi: \mathcal{G} \rightarrow \mathcal{G}_{C}$ is a group isomorphism.

The following three lemmas may be proved.

Lemma 3.3.1. If $\mathcal{G}_{C}$ acts reducibly on $C^{m}$ then $\mathcal{G}$ acts reducibly on $R^{n}$.

Let $C_{R}^{m}$ denote the vector space of $m$-tuples of complex numbers over the real field.

LEMma 3.3.2. $\mathcal{G}$ acts reducibly on $R^{n}$ if and only if $\mathcal{G}_{C}$ acts reducibly on $C_{R}^{m}$.

LEMMA 3.3.3. Let $\mathcal{H}$ be the subgroup of $G L(n ; R)$ whose elements $H$ satisfy

$$
\left\{\begin{array}{l}
{ }^{t} H K H=K, \text { and } \\
{ }^{t} H L H=L .
\end{array}\right.
$$

$\mathcal{H}$ is a subgroup of $\mathcal{F}$. Moreover, $\mathcal{H}_{C}=O(m ; C)$. 
3.4. TWO-DIMENSIONAL MANIFOLDS. We now turn our attention to the case $n=2$.

LEMMA 3.4.1. Let $\eta_{1}$ and $\eta_{2}$ be two independent, symmetric bilinear forms on $V$ of signature $(1,1)$. Let $\mathcal{G}$ be a subgroup of $\operatorname{Aut}(V)$ which preserves $\eta_{1}$ and $\eta_{2}$. Then $\mathcal{G}$ acts reducibly on $V$.

Proof: Suppose that $\mathcal{G}$ acts irreducibly on $V$. By Lemma 3.2.5, there exists a basis of $V$ in which $\eta_{1}$ and $\eta_{2}$ are represented, respectively, by $K$ and $a K+b L$, where $a, b \in R$. $\mathcal{G}$ may therefore be identified with a subgroup $\mathcal{H}$ of $G L(2 ; R)$ whose elements $H$ satisfy

$$
\left\{\begin{array}{l}
{ }^{t} H K H=K, \text { and } \\
{ }^{t} H L H=L .
\end{array} .\right.
$$

By Lemma 3.3.3, $\mathcal{H}$ may be identified with $\mathcal{H}_{C}$ and $\mathcal{H}_{C} \subseteq O(1 ; C)=\{-1,1\}$. Therefore $\mathcal{H} \subseteq\{-I, I\}$, which acts reducibly on $R^{2}$. Therefore $\mathcal{G}$ acts reducibly on $V$ and we obtain a contradiction.

This proves Theorem 2.0.1 (ii)

3.5. ThREe SyMmetric BILINEAR forms OF Signature $(m, m)$. Let $\eta_{1}, \eta_{2}$ and $\eta_{3}$ be three independent, symmetric bilinear forms on $V$ of signature $(m, m)$. Let $\mathcal{G}$ be a subgroup of $\operatorname{Aut}(V)$ which preserves $\eta_{1}, \eta_{2}$ and $\eta_{3}$. In this section we find normal forms for $\eta_{1}, \eta_{2}$ and $\eta_{3}$ when $\mathcal{G}$ acts irreducibly on $V$. Moreover, we show that if $m$ is odd then $\mathcal{G}$ acts reducibly on $V$.

LEMMA 3.5.1. Let $\eta_{1}, \eta_{2}$ and $\eta_{3}$ be three independent, symmetric bilinear forms on $V$ of signature $(m, m)$, where $n=2 m$. Let $\mathcal{G}$ be a subgroup of Aut $(V)$ whose elements preserve $\eta_{1}, \eta_{2}$ and $\eta_{3}$. Suppose that $\mathcal{G}$ acts irreducibly on $V$. Let $\beta$ be a basis of $V$ with respect to which $\eta_{1}$ and $\eta_{2}$ are represented, respectively, by $K$ and $a_{1} K+a_{2} L$, for some $a_{1}, a_{2} \in R$. With respect to $\beta, \eta_{3}$ is represented by $b_{1} K+b_{2} L+b_{3} M$ where $b_{1}, b_{2}, b_{3} \in R$, $(K M)^{2}=-I$ and $M$ has the form

$$
M=\left(\begin{array}{cc}
A & B \\
-B & A
\end{array}\right),
$$

where $A$ is symmetric and $B$ is skew-symmetric.

Proof: Let $\beta$ be a basis of $V$ with respect to which $\eta_{1}$ and $\eta_{2}$ are represented, respectively, by $K$ and $a_{1} K+a_{2} L$, for some $a_{1}, a_{2} \in R$. $\eta_{3}$ is represented by a symmetric matrix $N$. By Lemma 3.1.2, $N=b_{1} K+b_{2} K J$, where $b_{1}, b_{2} \in R$ and $J^{2}=-I$. $J$ has the form

$$
J=\left(\begin{array}{cc}
S & T \\
-{ }^{t} T & -U
\end{array}\right),
$$


where $S$ and $U$ are symmetric. $\mathcal{G}$ may be identified with a subgroup $\mathcal{H}$ of $G L(n ; R)$ whose elements $H$ satisfy

$$
\left\{\begin{array}{l}
{ }^{t} H K H=K, \\
{ }^{t} H L H=L, \text { and } \\
{ }^{t} H K J H=K J .
\end{array}\right.
$$

Let $H \in \mathcal{H}$. Then $H$ commutes with $K L, J$ and $L K J$.

By Lemma 3.1.1, the minimum polynomial $p=p(x)$ of $L K J$ is irreducible over $R$. Suppose $p$ were linear: $p(x)=x-a$, for some $a \in R$. Then $L K J=a I$. That is, $K J=a L$, which contradicts the independence of $\eta_{1}, \eta_{2}$ and $\eta_{3}$. Therefore the minimum polynomial of $L K J$ has degree 2: $p(x)=x^{2}+a x+b$, where $a, b \in R$.

The equations $p(L K J)=0$ and $J^{2}=-I$ give

$$
\left\{\begin{array}{ll}
b T+{ }^{t} T & =-a I, \\
T+b^{t} T & =-a I, \\
S & =b U, \text { and } \\
U & =b S .
\end{array}\right. \text {. }
$$

If $U=0$ then $S=U$. Suppose that $U \neq 0$. We have $U=b S=b^{2} U$. Consequently, $b= \pm 1$. Since $p(x)=x^{2}+a x+b$ is irreducible, $b=1$, and again $S=U$. Therefore in either case we have $S=U$.

The first two among the four equations above gives us $(1+b)\left(T+{ }^{t} T\right)=-2 a I$. Consequently $T+{ }^{t} T=2 c I$, where $c=-a /(1+b)$.

Set $E:=K J-c L$. $E$ is given by

$$
E=\left(\begin{array}{cc}
S & T^{\prime} \\
-T^{\prime} & S
\end{array}\right),
$$

where $S$ is symmetric and $T^{\prime}:=T-c I$ is skew-symmetric. Now $L K J+J L K=2 c I$. It follows that,

$$
(K E)^{2}=(J+c L K)^{2}=J^{2}+c(L K J+J L K)+c^{2}(L K)^{2}=-I+2 c^{2} I-c^{2} I=\left(c^{2}-1\right) I .
$$

The polynomial $q(x):=x^{2}+\left(1-c^{2}\right)$ annihilates $K E$. Since every element $H$ of $\mathcal{H}$ commutes both with $J$ and with $K L, H$ commutes with $K E$. By Lemma 3.1.1, the minimal polynomial of $K E$ is irreducible over $R$. Now, since $K, L$ and $K J$ are independent, $K E$ is not a multiple of the identity matrix. It follows that $q$ is the minimal polynomial of $K E$. Since $q$ is irreducible over $R, 1-c^{2}>0$. Now define the matrix $M$ by

$$
M:=\frac{E}{\sqrt{1-c^{2}}} \text {. }
$$

Then $(K M)^{2}=-I$. Moreover, $M$ has the required form. 
LEMMA 3.5.2. Let $\eta_{1}, \eta_{2}$ and $\eta_{3}$ be three independent, symmetric bilinear forms on $V$ of signature $(m, m)$, where $n=2 m$. Let $\mathcal{G}$ be a subgroup of Aut $(V)$ whose elements preserve $\eta_{1}, \eta_{2}$ and $\eta_{3}$. Suppose that $\mathcal{G}$ acts irreducibly on $V$. There exists a basis of $V$ with respect to which $\eta_{1}, \eta_{2}$ and $\eta_{3}$ are represented, respectively, by $K, a_{1} K+a_{2} L$ and $b_{1} K+b_{2} L+b_{3} M$, where $a_{1}, a_{2}, b_{1}, b_{2}, b_{3} \in R,(K M)^{2}=-I$ and $M$ has the form

$$
M=\left(\begin{array}{cc}
A & B \\
-B & D
\end{array}\right)
$$

where $D$ is diagonal and $B$ is skew-symmetric.

ProOF: Follows from Lemma 3.2.5, Lemma 3.5.1 and a standard diagonalisation argument.

Corollary 3.5.3. Let $\eta_{1}, \eta_{2}$ and $\eta_{3}$ be three independent, symmetric bilinear forms on $V$ of signature $(m, m)$, where $n=2 m$. Let $\mathcal{G}$ be a subgroup of Aut $(V)$ whose elements preserve $\eta_{1}, \eta_{2}$ and $\eta_{3}$. If $m$ is odd then $\mathcal{G}$ acts reducibly on $V$.

Proof: Suppose that $\mathcal{G}$ acts irreducibly on $V$ and that $m$ is odd. By Lemma 3.5.2, there exists a matrix $J=K M$ satisfying $J^{2}=-I$ and of the form

$$
J=\left(\begin{array}{cc}
D & B \\
B & -D
\end{array}\right)
$$

where $D$ is diagonal and $B$ is skew-symmetric. Since $B$ is a skew-symmetric $m \times m$ matrix with $m$ odd, we have $\operatorname{det} B=0$. On the other hand, we have the equation $D^{2}+I=-B^{2}$. Since $D$ is diagonal, $-B^{2}$ is diagonal, with positive entries along the main diagonal. Consequently, $\operatorname{det} B \neq 0$ and we obtain a contradiction. Therefore $\mathcal{G}$ acts reducibly on $V$.

This proves Theorem 2.0.1 (iii).

3.6. Four SyMMETRIC BILINEAR FORMS OF Signature $(m, m)$. Let $\eta_{1}, \eta_{2}, \eta_{3}$ and $\eta_{4}$ be four independent, symmetric bilinear forms on $V$ of signature $(m, m)$. Let $\mathcal{G}$ be a subgroup of $\operatorname{Aut}(V)$ which preserves $\eta_{1}, \eta_{2}, \eta_{3}$ and $\eta_{4}$. We shall show that $\mathcal{G}$ acts reducibly on $V$. Suppose therefore that $\mathcal{G}$ acts irreducibly on $V$. By Lemma 3.2.5 and Lemma 3.5.1, there exists a basis of $V$ in which $\eta_{1}, \eta_{2}, \eta_{3}$ and $\eta_{4}$ are represented, respectively, by $K, a_{1} K+a_{2} L, b_{1} K+b_{2} L+b_{3} M$ and $c_{1} K+c_{2} L+c_{3} N$, where $a_{j}, b_{j}, c_{j} \in R$, $(K M)^{2}=(K N)^{2}=-I$, and $M$ and $N$ are of the form

$$
M=\left(\begin{array}{cc}
P & Q \\
-Q & P
\end{array}\right) \text { and } N=\left(\begin{array}{cc}
S & T \\
-T & S
\end{array}\right)
$$


where $P$ and $S$ are symmetric and $Q$ and $T$ are skew-symmetric. $\mathcal{G}$ may be identified with a subgroup $\mathcal{H}$ of $G L(n ; R)$ whose elements $H$ satisfy

$$
\left\{\begin{array}{l}
{ }^{t} H K H=K, \\
{ }^{t} H L H=L, \\
{ }^{t} H M H=M, \text { and } \\
{ }^{t} H N H=N .
\end{array}\right.
$$

By Lemma 3.3.3, $\mathcal{H}$ may be identified with $\mathcal{H}_{C}$. Let $x:=\phi(M)$ and $y:=\phi(N)$.

LEMMA 3.6.1. ${ }^{t} x x={ }^{t} y y=-I_{m \times m}$.

Proof: We consider $\phi$ as an isomorphism $\phi: \mathcal{H} \rightarrow \mathcal{H}_{C}$. Since $P$ is symmetric and $Q$ is skew-symmetric, $K M K=\phi^{-1}\left({ }^{t} x\right)$. Now, $-I_{n \times n}=(K M)^{2}=(K M K) M=\phi^{-1}\left({ }^{t} x\right) M$. Therefore,

$$
-I_{m \times m}=\phi\left(-I_{n \times n}\right)=\phi\left(\phi^{-1}\left({ }^{t} x\right) M\right)=\phi\left(\phi^{-1}\left({ }^{t} x\right)\right) \phi(M)={ }^{t} x x .
$$

The proof for ${ }^{t} y y=-I_{m \times m}$ is similar.

It follows from Lemma 3.3.3, that $\mathcal{H}_{C}$ is a subgroup of $G L(m ; C)$ whose elements $h$ satisfy

$$
\left\{\begin{array}{l}
{ }^{t} h h=I, \\
{ }^{t} \bar{h} x h=x, \text { and } \\
{ }^{t} \bar{h} y h=y .
\end{array}\right.
$$

Now ${ }^{t} \bar{h}=x h^{-1} x^{-1}$. Therefore $y={ }^{t} \bar{h} y h=x h^{-1} x^{-1} y h$. Hence

$$
h\left(x^{-1} y\right)=\left(x^{-1} y\right) h,
$$

for all $h \in \mathcal{H}_{C}$. By Lemma 3.3.1, $\mathcal{H}_{C}$ acts irreducibly on $C^{m}$. By Lemma 3.1.1, the minimal polynomial for $x^{-1} y$ (over $C$ ) is irreducible (over $C$ ). Therefore $x^{-1} y=a I$, for some $a \in C$. That is, $y=a x$. By Lemma 3.6.1, $-I_{m \times m}={ }^{t} y y={ }^{t}(a x)(a x)=a^{2}\left({ }^{t} x x\right)$ $=-a^{2}$. Therefore $a= \pm 1$. Hence $N= \pm M$. This contradicts the independence of $\eta_{1}, \eta_{2}, \eta_{3}$ and $\eta_{4}$. Therefore $\mathcal{G}$ acts reducibly on $V$. This proves Theorem 2.0.1 (iv).

\section{ProOF OF THEOREM 2.0 .2}

In this section we provide examples of connections with irreducible holonomy and more than one parallel semi-Riemannian metric.

4.1. The CASE $n=2 m, m \geqslant 3$. Let $n=2 m$ and $m \geqslant 3$. Consider $S O(m ; C)$, the subgroup of $G L(m ; C)$ consisting of elements $h$ which satisfy

$$
\left\{\begin{array}{l}
{ }^{h} h=I, \text { and } \\
\operatorname{det} h=1 .
\end{array}\right.
$$


For $\xi \in C^{m}$, let $(\xi)$ denote the smallest $R$-subspace of $C_{R}^{m}$ containing $\xi$, which is $S O(m ; C)$-invariant. For two vectors $\xi_{1}, \xi_{2} \in C^{m}$ we let $\xi_{1} \cdot \xi_{2}=\sum_{k=1}^{m} \xi_{1}^{k} \xi_{2}^{k}$, denote the dot product. The norm of a vector $\xi \in R^{m}$ with respect to the dot product on $R^{m}$ will be denoted by $\|x i\|$. For $\lambda \in C$, set

$$
C_{\lambda}^{m}:=\left\{\xi \in C^{m} \mid \xi \cdot \xi=\lambda\right\} .
$$

Lemma 4.1.1. Let $m \geqslant 3$. For $0 \neq \xi \in C_{0}^{m},(\xi) \nsubseteq C_{0}^{m}$.

Proof: Let $0 \neq \xi \in C_{0}^{m}$. Then with $\xi=\xi_{R}+i \xi_{I}$, where $\xi_{R}, \xi_{I} \in R^{m}$,

$$
\begin{cases}\xi_{R} \cdot \xi_{R}-\xi_{I} \cdot \xi_{I} & =0, \text { and } \\ \xi_{R} \cdot \xi_{I} & =0 .\end{cases}
$$

Therefore $\xi_{R} \neq 0 \neq \xi_{I}$. Since $m \geqslant 3$, we may choose $h \in S O(m ; R) \subset S O(m ; C)$ such that

$$
h \xi_{R}=\kappa e_{1} \text { and } h \xi_{I}=\kappa e_{2},
$$

where $\kappa:=\left\|\xi_{R}\right\|=\left\|\xi_{I}\right\|$. Here $e_{j}$ denotes the $j^{\text {th }}$ vector of the standard basis of $R^{m}$. We have $h \xi=\kappa\left(e_{1}+i e_{2}\right)$. Let $g \in S O(m ; R)$ be the element defined by

$$
g:=\left(\begin{array}{ccc|c}
0 & 1 & 0 & \\
1 & 0 & 0 & 0 \\
0 & 0 & -1 & \\
\hline 0 & & I
\end{array}\right),
$$

where $I$ is the $(m-3) \times(m-3)$ identity matrix. Now $g h \xi=\kappa\left(i e_{1}+e_{2}\right)$. Hence $\kappa(1+i)\left(e_{1}+e_{2}\right)=(1+g) h \xi \in(\xi)$. Moreover, $\kappa(1+i)\left(e_{1}+e_{2}\right) \notin C_{0}^{m}$.

LEMma 4.1.2. Let $0 \neq \lambda \in C$. $S O(m ; C)$ acts transitively on $C_{\lambda}^{m}$.

Proposition 4.1.3. Let $m \geqslant 3 . S O(m ; C)$ acts irreducibly on $C_{R}^{m}$.

Proof: Let $\xi \in C^{m}$. By Lemma 4.1.1, there exists $\xi^{\prime} \in(\xi)$, where $\xi^{\prime} \in C_{\lambda}^{m}$ and $\lambda \neq 0$. Let $\left\{\xi_{1}, \ldots, \xi_{n}\right\}$ be a basis of $C_{R}^{m}$ for which $\xi_{j} \in C_{\lambda}^{m}$, for each $1 \leqslant j \leqslant n$. By Lemma $4.1 .2, \xi_{j} \in(\xi)$ for all $1 \leqslant j \leqslant n$, and so $(\xi)=C_{R}^{m}$.

Let $\mathcal{H}_{n}$ denote the subgroup of $G L(n ; R)$ whose elements $H$ satisfy

$$
\left\{\begin{array}{l}
{ }^{t} H K H=K, \text { and } \\
{ }^{t} H L H=L .
\end{array}\right.
$$

Let $\mathcal{H}_{n}^{0}$ be the identity component of $\mathcal{H}_{n}$. By Lemma 3.3.3, we have a group isomorphism $\phi: \mathcal{H}_{n} \rightarrow O(m ; C)$. The identity component of $O(m ; C)$ is $S O(m ; C)$ and so $\phi: \mathcal{H}_{n}^{0}$ $\rightarrow S O(m ; C)$ is an isomorphism of groups. 
COROLlary 4.1.4. Let $m \geqslant 3 . \mathcal{H}_{n}^{0}$ acts irreducibly on $R^{n}$.

Proof: This follows from Lemma 3.3.2 and Proposition 4.1.3.

We are now able to demonstrate the existence of connections with irreducible holonomy that have two independent, parallel semi-Riemannian metrics. Let $\theta$ be a generic $n \times n$ matrix of 1 -forms on $R^{n}$ that takes its values in $\mathbf{h}_{n}$, the Lie algebra of $\mathcal{H}_{n}$. Define the connection $\nabla$ on $R^{n}$ by

$$
\nabla_{X_{i}} d x^{j}:=-\sum_{l=1}^{n} \theta_{l}^{j}\left(X_{i}\right) d x^{l}
$$

where $X_{i}$ denotes the vector field $\partial / \partial x^{i}$, for $1 \leqslant i \leqslant n$. The curvature form with respect to the moving frame $\left(X_{1}, \ldots, X_{n}\right)$ is given by $\Theta=d \theta+\theta \wedge \theta$. Owing to the genericity of $\theta$, the Lie algebra of the infinitesimal holonomy group of $\nabla$, at each $x \in R^{n}$, is $\mathbf{h}_{n}$. Therefore the infinitesimal holonomy group of $\nabla$ at $x$ contains a subgroup isomorphic to $\mathcal{H}_{n}^{0}$ and so acts irreducibly on $T_{x} R^{n}$, by Corollary 4.1.4.

Let $g_{1}$ and $g_{2}$ be, respectively, the metrics on $R^{n}$ represented by $K$ and $L$ with respect to the moving frame $\left(X_{1}, \ldots, X_{n}\right)$. We have

$$
\left\{\begin{array}{l}
K \theta+{ }^{t} \theta K=0, \text { and } \\
L \theta+{ }^{t} \theta L=0 .
\end{array}\right.
$$

A metric $g=\sum_{i, j=1}^{n} C_{i j} d x^{i} \otimes d x^{j}$, where $C \in G L(n ; R)$, is parallel with respect to $\nabla$ if and only if $C \theta+{ }^{i} \theta C=0$, and so $g_{1}$ and $g_{2}$ are parallel metrics. This proves Theorem 2.0.2 (i).

4.2. THE CASE $n=4 r, r \geqslant 2$. Let $n=2 m$, as before, and let $m=2 r$ and $r \geqslant 2$. Define $M \in G L(n ; R)$ by

$$
M:=\left(\begin{array}{cc}
0 & T \\
-T & 0
\end{array}\right),
$$

where $T \in G L(m ; R)$ is given by

$$
T:=\left(\begin{array}{cc}
0 & I_{r \times r} \\
-I_{r \times r} & 0
\end{array}\right) .
$$

Note that $\phi(M)=i T$. Let $\mathcal{F}_{n}$ denote the subgroup of $G L(n ; R)$ whose elements $F$ satisfy

$$
\left\{\begin{array}{l}
{ }^{t} F K F=K, \\
{ }^{t} F L F=L, \text { and } \\
{ }^{t} F M F=M .
\end{array}\right.
$$


By Lemma 3.3.3, $\mathcal{F}_{n}$ may be identified with the subgroup $\mathcal{G}_{m}$ of $G L(m ; C)$ whose elements $g$ satisfy

$$
\left\{\begin{array}{l}
{ }^{t} g g=I, \text { and } \\
\bar{g} T=T g .
\end{array}\right.
$$

Now $g$ is an element of $\mathcal{G}_{m}$ if and only if ${ }^{t} g g=I$ and it has the form

$$
g=\left(\begin{array}{cc}
P & Q \\
-\bar{Q} & \bar{P}
\end{array}\right) .
$$

Let $\mathcal{F}_{n}^{0}$ denote the identity component of $\mathcal{F}_{n}$ and let $\mathcal{G}_{m}^{0}$ denote the identity component of $\mathcal{G}_{m}$. $\phi: \mathcal{F}_{n}^{0} \rightarrow \mathcal{G}_{m}^{0}$ is a group isomorphism. Denote by $\mathbf{g}_{m}$ the Lie algebra of $\mathcal{G}_{m}$. $D \in g l(m ; C)$ is an element of $\mathbf{g}_{m}$ if and only if it has the form

$$
D=\left(\begin{array}{cc}
A & B \\
-\bar{B} & \bar{A}
\end{array}\right),
$$

where $A=-{ }^{t} A$ and $B={ }^{t} \bar{B}$.

LEMMA 4.2.1. Let $\mathcal{H}$ be a Lie subgroup of $G L(m ; C)$ and let $\mathbf{h}$ be the corresponding Lie algebra. $\mathcal{H}$ and $\mathrm{h}$ act on $C_{R}^{m}$ on the left. If $W$ is an $\mathcal{H}$-invariant subspace of $C_{R}^{m}$ then it is also $\mathrm{h}$-invariant.

Let $\{0\} \neq W \subseteq C_{R}^{m}$ be a $\mathcal{G}_{m}^{0}$-invariant subspace (over $R$ ). By Lemma 4.2.1, $W$ is also $\mathbf{g}_{m}$-invariant. Using Proposition 4.1.3 and the form for elements of $\mathcal{G}_{m}$ and $g_{m}$ given above one can demonstrate the following lemma.

LEMMA 4.2.2.

(1) There exist elements

$$
\left(\begin{array}{c}
X_{1} \\
Y_{1}
\end{array}\right), \ldots,\left(\begin{array}{c}
X_{m} \\
Y_{m}
\end{array}\right) \in W
$$

such that $X_{1}, \ldots, X_{m} \in C^{r}$ are $R$-linearly independent.

(2) There exist elements

$$
\left(\begin{array}{l}
U_{1} \\
V_{1}
\end{array}\right), \ldots,\left(\begin{array}{l}
U_{m} \\
V_{m}
\end{array}\right) \in W
$$

such that $V_{1}, \ldots, V_{m} \in C^{r}$ are $R$-linearly independent. Moreover, if

$$
\left(\begin{array}{c}
X \\
0
\end{array}\right) \in W \text { or }\left(\begin{array}{l}
0 \\
X
\end{array}\right) \in W
$$

for some $0 \neq X \in C^{r}$, then we may take $Y_{1}=\cdots=Y_{m}=0$ and $U_{1}=\cdots=U_{m}=0$.

COROLlary 4.2.3. If

$$
\left(\begin{array}{l}
X \\
0
\end{array}\right) \in W \text { or }\left(\begin{array}{l}
0 \\
X
\end{array}\right) \in W,
$$

for some $0 \neq X \in C^{r}$, then $W=C_{R}^{m}$. 
CoRollary 4.2.4. If $W \neq C_{R}^{m}$, then $\operatorname{dim}_{R} W=m$.

Proof: Suppose that $\operatorname{dim}_{R} W>m$. Then there exist elements

$$
\left(\begin{array}{c}
X_{1} \\
Y_{1}
\end{array}\right), \ldots,\left(\begin{array}{c}
X_{m+1} \\
Y_{m+1}
\end{array}\right) \in W
$$

which are linearly independent over $R$. Let $a^{j} \in R, 1 \leqslant j \leqslant m+1$, be such that $\sum_{j=1}^{m+1} a^{j} Y_{j}=0$ and $a^{l} \neq 0$ for some $l \in\{1, \ldots, m+1\}$. We must have $X:=\sum_{j=1}^{m+1} a^{j} X_{j} \neq 0$. Now

$$
\left(\begin{array}{c}
X \\
0
\end{array}\right)=\sum_{j=1}^{m+1} a^{j}\left(\begin{array}{c}
X_{j} \\
Y_{j}
\end{array}\right) \in W
$$

By Corollary 4.2.3, $W=C_{R}^{m}$, which contradicts our hypothesis. Consequently, $\operatorname{dim}_{R} W$ $\leqslant m$. By Lemma 4.2.2, $\operatorname{dim}_{R} W \geqslant m$ and so we have $\operatorname{dim}_{R} W=m$.

Corollary 4.2.5. Suppose that $W \neq C_{R}^{m}$. Let $Y \in C^{r}$. Then there exists a unique $X \in C^{r}$ such that

$$
\left(\begin{array}{l}
X \\
Y
\end{array}\right) \in W
$$

Proof: Existence follows from Lemma 4.2.2 and uniqueness from Corollary 4.2.3. Henceforth we shall suppose that $W \neq C_{R}^{m}$. This will lead to a contradiction.

Let $s, t \in\{1, \ldots, r\}$. We let $\xi_{s, t}$ denote the unique element in $C^{r}$ such that

$$
\left(\begin{array}{c}
\xi_{s, t} \\
e_{s}+e_{t}
\end{array}\right) \in W
$$

and we let $\xi_{s, t}^{\prime}$ denote the unique element in $C^{r}$ such that

$$
\left(\begin{array}{c}
\xi_{s, t}^{\prime} \\
i\left(e_{s}+e_{t}\right)
\end{array}\right) \in W
$$

where $e_{s}, e_{t}$ are standard basis vectors in $R^{r}$ (see Corollary 4.2.5.)

LEMMA 4.2.6.

(1) $\xi_{s, t}=a_{s, t}\left(e_{s}+e_{t}\right)$, for some $a_{s, t} \in C$.

(2) $\xi_{s, t}^{\prime}=b_{s, t} i\left(e_{s}+e_{t}\right)$, for some $b_{s, t} \in C$.

Furthermore, using Corollary 4.2.5 and Lemma 4.2.6 we can prove the following.

LEMMA 4.2.7. $a_{s, t}=-b_{s, t}$ for $1 \leqslant s \neq t \leqslant r$.

Now let $Y_{1}:=e_{1}+e_{2} \in R^{r}$. Extend $Y_{1}$ to a real basis $Y_{1}, \ldots, Y_{r}$ of $R^{r}$ over $R$. Then $Y_{1}, \ldots, Y_{r}, i Y_{1}, \ldots, i Y_{r}$ is a basis of $C_{R}^{r}$. By Corollary 4.2.4 and Corollary 4.2.5, there exist unique $X_{1}, \ldots, X_{r}, X_{1}^{\prime}, \ldots, X_{r}^{\prime} \in C^{r}$, such that

$$
\beta:=\left\{\left(\begin{array}{c}
X_{1} \\
Y_{1}
\end{array}\right), \ldots,\left(\begin{array}{c}
X_{r} \\
Y_{r}
\end{array}\right),\left(\begin{array}{c}
X_{1}^{\prime} \\
i Y_{1}
\end{array}\right), \ldots,\left(\begin{array}{c}
X_{r}^{\prime} \\
i Y_{r}
\end{array}\right)\right\}
$$


is a basis for $W$ over $R$. For convenience, set $c:=a_{1,2}$. Then $X_{1}=c Y_{1}$ and $X_{1}^{\prime}=-c i Y_{1}$, by Lemma 4.2.6 and Lemma 4.2.7. Now

$$
\left(\begin{array}{c}
-Y_{1} \\
c Y_{1}
\end{array}\right)=\left(\begin{array}{cc}
0 & -I \\
I & 0
\end{array}\right)\left(\begin{array}{c}
c Y_{1} \\
Y_{1}
\end{array}\right) \in W
$$

Therefore,

$$
\left(\begin{array}{c}
-Y_{1} \\
c Y_{1}
\end{array}\right)
$$

must be a real, linear combination of elements in the basis $\beta$. That is, there exists $a^{j}, b^{j} \in R, 1 \leqslant j \leqslant r$, such that

$$
\left(\begin{array}{c}
-Y_{1} \\
c Y_{1}
\end{array}\right)=\sum_{j=1}^{r}\left(a^{j}\left(\begin{array}{c}
X_{j} \\
Y_{j}
\end{array}\right)+b^{j}\left(\begin{array}{c}
X_{j}^{\prime} \\
i Y_{j}
\end{array}\right)\right)
$$

In particular, $c Y_{1}=\sum_{j=1}^{r}\left(a^{j}+i b^{j}\right) Y_{j}$. Since $Y_{1}, \ldots, Y_{r} \in R^{r}$ is a basis of $R^{r}$ over $R$, it is a basis of $C^{r}$ over $C$. It follows therefore, that $c=a^{1}+i b^{1}$ and $a^{j}=b^{j}=0$, for $2 \leqslant j \leqslant r$. Hence $c_{R}=a^{1}$ and $c_{I}=b^{1}$, where $c_{R}$ and $c_{I}$ denote the real and imaginary parts of $c$, respectively. Now,

$$
-Y_{1}=\sum_{j=1}^{r}\left(a^{j} X_{j}+b^{j} X_{j}^{\prime}\right)=\|c\|^{2} Y_{1},
$$

where \|\| denotes the norm of the standard inner product on $C^{r}$. Thus $\|c\|^{2}=-1$, which is a contradiction. Therefore $W=C_{R}^{m}$. We have shown the following.

PROPOSITION 4.2.8. $\mathcal{G}_{m}^{0}$ acts irreducibly on $C_{R}^{m}$.

Corollary 4.2.9. $\mathcal{F}_{n}^{0}$ acts irreducibly on $R^{n}$.

PROof: The corollary follows from Proposition 4.2.8 and Lemma 3.3.2.

A linear connection with irreducible holonomy group that has three parallel semiRiemannian metrics can be constructed in a manner analogous to the prior case with two parallel metrics described at the end of Subsection 4.1. This demonstrates Theorem 2.0 .2 (ii).

\section{Four-Dimensional Manifolds}

5.1. Simply CONNECTEd Four-Dimensional manifolds. We have seen in Proposition 4.1.3 that for $m \geqslant 3, S O(m ; C)$ acts irreducibly on $C_{R}^{m}$. By contrast, for the case $m=2$, we have the following.

LEMMA 5.1.1. $S O(2 ; C)$ acts reducibly on $C_{R}^{2}$.

Let $g_{1}$ and $g_{2}$ be two independent semi-Riemannian metrics of signature $(2,2)$ on a connected, simply connected four-dimensional manifold $M$, parallel with respect to a 
linear connection $\nabla$ on $M$, and let $\mathcal{G}$ denote the holonomy group of $\nabla$ based at some point $m \in M$. Suppose that $\mathcal{G}$ acts irreducibly on $T_{m} M$. By Lemma 3.2.5, there exists a basis $\beta$ of $T_{m} M$ in which $g_{1}(m)$ and $g_{2}(m)$ are represented, respectively, by $K$ and $a K+b L$, where $a, b \in R$. $\mathcal{G}$ may be identified with a subgroup $\mathcal{H}$ of $G L(4 ; R)$ whose elements $H$ satisfy

$$
\left\{\begin{array}{l}
{ }^{t} H K H=K, \text { and } \\
{ }^{t} H L H=L .
\end{array}\right.
$$

By Lemma 3.3.3, $\mathcal{H}$ may be identified with $\mathcal{H}_{C}$, a subgroup of $O(2 ; C)$. However, the holonomy group of a connection on a connected, simply connected manifold consists of only one component (see [5, p. 73]). Therefore $\mathcal{H}_{C}$ is in fact a subgroup of $S O(2 ; C)$. By Lemma 5.1.1, $\mathcal{H}_{C}$ acts reducibly on $C_{R}^{2}$. It now follows from Lemma 3.3.2 that $\mathcal{H}$ acts reducibly on $R^{4}$ contradicting the assumption that $\mathcal{G}$ acts irreducibly on $T_{m} M$. This, along with Lemma 3.1.3, proves Theorem 2.0.3 (i).

5.2. Three SyMmetric Bilinear Forms OF Signature $(2,2)$. Let $\eta_{1}, \eta_{2}$ and $\eta_{3}$ be three independent, symmetric bilinear forms on $V$ of signature $(2,2)$. Let $\mathcal{G}$ be a subgroup of Aut $(V)$ which preserves $\eta_{1}, \eta_{2}$ and $\eta_{3}$. Suppose that $\mathcal{G}$ acts irreducibly on $V$. We shall obtain a contradiction. By Lemma 3.5.2, there exists a basis $\beta$ of $V$ in which $\eta_{1}, \eta_{2}$ and $\eta_{3}$ are represented respectively by $K, a_{1} K+a_{2} L$ and $b_{1} K+b_{2} L+b_{3} M$, where $(K M)^{2}=-I$ and $M$ has the form

$$
M:=\left(\begin{array}{cc}
D & S \\
-S & D
\end{array}\right)
$$

where $D$ is diagonal and $S$ is skew-symmetric. $\mathcal{G}$ may be identified with a subgroup $\mathcal{H}$ of $G L(4 ; R)$ whose elements satisfy

$$
\left\{\begin{array}{l}
{ }^{t} H K H=K, \\
{ }^{t} H L H=L, \text { and } \\
{ }^{t} H M H=M .
\end{array}\right.
$$

Let $x:=\phi(M)=D+i S$. In light of Lemma 3.3.3, $\mathcal{H}$ may be identified with $\mathcal{H}_{C}$, a subgroup of $G L(2 ; C)$ whose elements $h$ satisfy

$$
\left\{\begin{array}{l}
{ }^{t} h h=I, \text { and } \\
\bar{h} x=x h .
\end{array}\right.
$$

Set

$$
D=\left(\begin{array}{ll}
p & 0 \\
0 & q
\end{array}\right) \text { and } S=s\left(\begin{array}{cc}
0 & 1 \\
-1 & 0
\end{array}\right)
$$


where $p, q, s \in R$. Now $(K M)^{2}=-I$ gives us

$$
\left\{\begin{array}{l}
p^{2}-s^{2}=-1, \\
q^{2}-s^{2}=-1, \text { and } \\
s(p-q)=0
\end{array}\right.
$$

$s=0$ implies $p^{2}=-1$ which would be a contradiction. Hence $s \neq 0$ and so $p=q$. We therefore have

$$
x=\left(\begin{array}{cc}
p & i s \\
-i s & p
\end{array}\right),
$$

where $s^{2}=p^{2}+1$. Let $h \in \mathcal{H}_{C}$. Since ${ }^{t} h h=I, h$ has one of the following two forms:

$$
\text { (1) } h=\left(\begin{array}{cc}
a & b \\
-b & a
\end{array}\right) \text { or } \quad \text { (2) } h=\left(\begin{array}{cc}
a & b \\
b & -a
\end{array}\right) \text {, }
$$

where, in either case, $a^{2}+b^{2}=1$, for $a, b \in C$.

CASE 1. Suppose that $h \in \mathcal{H}_{C}$ has the form given by (1). The equation $\bar{h} x=x h$ is equivalent to

$$
\left\{\begin{array}{l}
i p a_{I}+s b_{I}=0, \text { and } \\
s a_{I}-i p b_{I}=0 .
\end{array}\right.
$$

It follows that $a_{I}=b_{I}=0$. That is, $a, b \in R$. Therefore $h \in \mathcal{H}_{C}$ has the form (1) if and only if $h \in S O(2 ; R)$.

CAse 2. Suppose that $h \in \mathcal{H}_{C}$ has the form given by (2). The equation $\bar{h} x=x h$ is equivalent to

$$
\left\{\begin{array}{l}
p a_{I}+s b_{R}=0, \text { and } \\
s a_{R}-p b_{I}=0 .
\end{array}\right.
$$

Hence $p^{2}=p^{2}\left(a^{2}+b^{2}\right)=-\left(a_{R}^{2}+b_{R}^{2}\right)$, and so $a_{R}=b_{R}=0$. However, this contradicts the requirement $a^{2}+b^{2}=1$. Therefore there are no elements $h \in \mathcal{H}_{C}$ of the form (2).

We have shown that $\mathcal{H}_{C}$ is a subgroup of $S O(2 ; R)$. By Lemma $5.1 .1, \mathcal{H}_{C}$ acts reducibly on $C_{R}^{2}$. By Lemma 3.3.2, $\mathcal{H}$ acts reducibly on $R^{4}$ contradicting the assumption that $\mathcal{G}$ acts irreducibly on $V$. We have shown the following corollary.

Corollary 5.2.1. Let $\eta_{1}, \eta_{2}$ and $\eta_{3}$ be three independent, symmetric bilinear forms on $V$ of signature $(2,2)$. Let $\mathcal{G}$ be a subgroup of Aut $(V)$ which preserves $\eta_{1}, \eta_{2}$ and $\eta_{3}$. Then $\mathcal{G}$ acts reducibly on $V$.

Theorem 2.0.3 (ii) follows from Lemma 3.1.3 and Corollary 5.2.1. 
5.3. IRREDUCIBILITY OF THE HOLONOMY GROUP. In this section we shall construct an example of a (non-simply connected) four-dimensional manifold endowed with two independent semi-Riemannian metrics of signature $(2,2)$, which are parallel with respect to a linear connection having an irreducible holonomy group.

Let $G \in G L(4 ; R)$ be defined by

$$
G:=\left(\begin{array}{cc|cc}
\sqrt{2} & 0 & 0 & 1 \\
0 & -\sqrt{2} & 1 & 0 \\
\hline 0 & -1 & \sqrt{2} & 0 \\
-1 & 0 & 0 & -\sqrt{2}
\end{array}\right),
$$

and define $g \in G L(2 ; C)$ by $g:=\phi(G)$. Now $g^{2}=I={ }^{t} g g$, and so by Lemma 3.3.3, we have

$$
\left\{\begin{array}{l}
G^{2}=I, \\
{ }^{t} G K G=K, \text { and } \\
{ }^{t} G L G=L .
\end{array}\right.
$$

Let $\mathcal{G}$ denote the group consisting of the two elements $I$ and $G: \mathcal{G}:=\{I, G\}$. The characteristic polynomial $p$ of $G$ is given by $p(x)=\left(x^{2}-1\right)^{2}$. The eigenspace $E$ of $G$ corresponding to the eigenvalue $\lambda=1$ is a two-dimensional subspace of $R^{4}$. Set $M^{\prime}:=R^{4}-E . \mathcal{G}$ acts properly discontinously (see [5, Vol. I, pp. 43-44]) on the left of $M^{\prime}$, by matrix multiplication. Let $g_{1}$ and $g_{2}$ denote the semi-Riemannian metrics on $M^{\prime}$ represented, respectively, by $K$ and $L$ in the moving frame $\left(d x^{1}, \ldots, d x^{4}\right)$ on $M^{\prime}$. We see that $L_{G}^{*}\left(g_{i}\right)=g_{i}$, for $i=1,2$, where $L_{G}$ denotes left multiplication by $G$.

Define the 1-forms $\alpha$ and $\beta$ on $M^{\prime}$ by

$$
\alpha:=x^{1} d x^{4}-x^{4} d x^{1} \text { and } \beta:=x^{2} d x^{3}-x^{3} d x^{2} .
$$

Define the matrix of 1-forms $\theta$ by

$$
\theta:=\left(\begin{array}{cc|cc}
0 & \alpha & 0 & \beta \\
-\alpha & 0 & -\beta & 0 \\
\hline 0 & -\beta & 0 & \alpha \\
\beta & 0 & -\alpha & 0
\end{array}\right) .
$$

The following two lemmas may be demonstrated by a direct calculation.

LEMMA 5.3.1. $G \theta=-\theta G$.

LEMMA 5.3.2. $L_{G}^{*}(\theta)=-\theta$.

Define the linear connection $\nabla$ on $M^{\prime}$ by

$$
\nabla_{X_{i}} d x^{j}:=-\sum_{l=1}^{4} \theta_{l}^{j}\left(X_{i}\right) d x^{l}
$$

for $1 \leqslant i, j \leqslant 4$, where $X_{i}=\partial / \partial x^{i}$. 
LEMMA 5.3.3. $g_{1}$ and $g_{2}$ are parallel with respect to $\nabla$.

ProOF: A metric $g=\sum_{i, j=1}^{4} C_{i j} d x^{i} \otimes d x^{j}$ on $M^{\prime}$, with $C \in G L(4 ; R)$, is parallel with respect to $\nabla$ if and only if $C \theta+{ }^{t} \theta C=0$. The lemma follows from the fact that $K \theta+{ }^{t} \theta K=0$ and $L \theta+{ }^{t} \theta L=0$.

For a vector field $X$ on $M^{\prime}$, we let $L_{G}(X)$ denote the push-forward of $X$ :

$$
L_{G}(X)(x):=L_{G *}\left(X\left(L_{G}(x)\right)\right)
$$

for $x \in M^{\prime}$.

LEMMA 5.3.4. Let $X, Y$ be vector fields on $M^{\prime}$. Then

$$
L_{G}\left(\nabla_{X} Y\right)=\nabla_{L_{G}(X)} L_{G}(Y)
$$

Proof: It suffices to consider $X=X_{i}=\partial / \partial x^{i}$ and $Y=X_{j}=\partial / \partial x^{j}$.

$$
\begin{aligned}
L_{G}\left(\nabla_{X_{i}} X_{j}\right)(x) & =\sum_{s=1}^{4} \sum_{t=1}^{4} G_{s}^{t} \theta_{j}^{s}\left(\left.X_{i}\right|_{G x}\right) X_{t}(x) \\
& =-\sum_{s=1}^{4} \sum_{t=1}^{4} G_{j}^{s} \theta_{s}^{t}\left(\left.X_{i}\right|_{G x}\right) X_{t}(x), \quad \text { by Lemma } 5.3 .1 \\
& =\sum_{s=1}^{4} \sum_{t=1}^{4} G_{j}^{s} L_{G}^{*}\left(\theta_{s}^{t}\right)\left(\left.X_{i}\right|_{G x}\right) X_{t}(x), \quad \text { by Lemma 5.3.2 } \\
& =\nabla_{L_{G}\left(X_{i}\right)} L_{G}\left(X_{j}\right)(x) .
\end{aligned}
$$

In summary, the left group action of $\mathcal{G}$ on $M^{\prime}$ preserves $g_{1}, g_{2}$ and $\nabla$. It follows that $g_{1}, g_{2}$ and $\nabla$ project to the quotient manifold

$$
M:=M^{\prime} / \mathcal{G}
$$

We denote the projections of $g_{1}, g_{2}$ and $\nabla$ to $M$ by the same symbols.

Now let $\gamma:[0,1] \rightarrow M^{\prime}$ be the smooth curve defined by

$$
\gamma(t):=\left(\begin{array}{c}
1-2 t \\
t(t-1) \\
t(t-1) \\
(\sqrt{2}+1)(2 t-1)
\end{array}\right)
$$

Set $x_{0}:=\gamma(0)$ and $x_{1}:=\gamma(1)$. Since $x_{1}=G x_{0}, \gamma$ is a closed loop in $M$. Let $\tau^{\prime}$ : $T_{x_{0}} M^{\prime} \rightarrow T_{x_{1}} M^{\prime}$ denote parallel translation along $\gamma$ in $M^{\prime}$ and let $\tau: T_{x_{0}} M \rightarrow T_{x_{0}} M$ denote parallel translation along $\gamma$ in $M$. Let $\left.a^{j} X_{j}\right|_{x_{0}} \in T_{x_{0}} M^{\prime}$, where we have used 
the summation convention. Then $\tau^{\prime}\left(\left.a^{j} X_{j}\right|_{x_{0}}\right)=\left.f^{j}(1) X_{j}\right|_{x_{1}}$, where $f:[0,1] \rightarrow R^{4}$ is the unique curve satisfying

$$
\begin{cases}\dot{f}+\theta(\dot{\gamma}) f=0, & \text { and } \\ f^{j}(0)=a^{j}, & 1 \leqslant j \leqslant 4 .\end{cases}
$$

Now $\theta(\dot{\gamma}) \equiv 0$ and so $f=f(t)$ is constant. Therefore $\tau^{\prime}\left(\left.a^{j} X_{j}\right|_{x_{0}}\right)=\left.a^{j} X_{j}\right|_{x_{1}}$. It follows that

$$
\tau\left(\left.a^{j} X_{j}\right|_{x_{0}}\right)=\left.G_{k}^{j} a^{k} X_{j}\right|_{x_{0}} .
$$

We identify $T_{x_{0}} M$ with $R^{4}$ by means of the basis $\left(X_{1}, \ldots, X_{4}\right)$ and hence the holonomy group $\Psi\left(x_{0}\right)$ of $\nabla$ at $x_{0} \in M$ is identified with elements of $G L(4 ; R)$. We have shown above that $G \in \Psi\left(x_{0}\right)$.

The curvature form $\Omega$ of $\nabla$ is

$$
\Omega=d \theta+\theta \wedge \theta=d \theta=\left(\begin{array}{cc|cc}
0 & d \alpha & 0 & d \beta \\
-d \alpha & 0 & -d \beta & 0 \\
\hline 0 & -d \beta & 0 & d \alpha \\
d \beta & 0 & -d \alpha & 0
\end{array}\right)
$$

Now $d \alpha=2 d x^{1} \wedge d x^{4}$ and $d \beta=2 d x^{2} \wedge d x^{3}$. The Lie algebra $\psi^{\text {inf }}\left(x_{0}\right)$ of the infinitesimal holonomy group $\Psi^{\text {inf }}\left(x_{0}\right)$ of $\nabla$ at $x_{0} \in M$ consists of elements of the form

$$
S:=\left(\begin{array}{cc|cc}
0 & a & 0 & b \\
-a & 0 & -b & 0 \\
\hline 0 & -b & 0 & a \\
b & 0 & -a & 0
\end{array}\right)
$$

where $a, b \in R$. The Lie algebra $\psi_{C}^{\text {inf }}\left(x_{0}\right)$ of the complexified infinitesimal holonomy group $\Psi_{C}^{\text {inf }}\left(x_{0}\right)$ consists of elements of the form

$$
s=\left(\begin{array}{cc}
0 & a \\
-a & 0
\end{array}\right),
$$

where $a \in C$. That is, $\psi_{C}^{\text {inf }}\left(x_{0}\right)=s o(2 ; C)$ and so $\Psi_{C}^{\text {inf }}\left(x_{0}\right)=S O(2 ; C)$.

Let $\mathcal{A}$ denote the $R$-subalgebra of $g l(2 ; C)$ generated by the matrices $g$ and $S O(2 ; C)$ over $R$. It is not difficult to demonstrate the following lemma.

LeMma 5.3.5. $\mathcal{A}=g l(2 ; C)$.

Let $\mathcal{H}$ be the subgroup of $G L(4 ; R)$ such that $\mathcal{H}_{C}$ is the subgroup of $G L(2 ; C)$ generated by $g$ and $S O(2 ; C)$. By Lemma $5.3 .5, \mathcal{H}_{C}$ acts irreducibly on $C_{R}^{2}$. By Lemma 3.3.2, $\mathcal{H}$ acts irreducibly on $R^{4}$. Since $\mathcal{H} \subseteq \Psi\left(x_{0}\right), \Psi\left(x_{0}\right)$ acts irreducibly on $T_{x_{0}} M$. This proves Theorem 2.0.3 (iii). 


\section{REFERENCES}

[1] S.K. Donaldson, 'An application of gauge theory to the topology of 4-manifolds', J. Differential Geom. 18 (1983), p. 279-315.

[2] M. Freedman, 'There is no room to spare in four dimensional space', Notices Amer. Math. Soc. 31 (1984), 3-6.

[3] G.S. Hall and C.B.G. McIntosh, 'Algebraic determination of the metric from the curvature in general relativity', Internat. J. Theoret. Phys. 22 (1983), 469-476.

[4] E. Ihrig, 'An exact determination of the gravitational potentials $g_{i j}$ in terms of the gravitational fields $R_{i j k}^{l}, J$. Math. Phys. 16 (1975), 54-55.

[5] S. Kobayashi and K. Nomizu, Foundations of differential geometry, Volume I (Wiley, New York, 1963).

[6] G.K. Martin and G. Thompson, 'Non-uniqueness of the metric in Lorentzian manifolds', Pacific J. Math. 158 (1993), 177-187.

[7] T.J. Willmore, An introduction to differential geometry (Clarendon Press, Oxford, 1959).

Faculty of Natural and Applied Sciences

Trinity Western University

7600 Glover Road

Langley, BC, V2Y $1 Y 1$

Canada

e-mail: richard.atkins@twu.ca 